\title{
Hyperacute multi-organ thromboembolic storm in COVID-19: a case report
}

\author{
Sarah Chibane ${ }^{1} \cdot$ Guillaume Gibeau $^{2} \cdot$ Frédéric Poulin $^{2} \cdot$ Pierre Tessier $^{2} \cdot$ Michelle Goulet $^{2} \cdot$ Marc Carrier $^{3}$. \\ Sylvain Lanthier ${ }^{4}$ D
}

Published online: 6 June 2020

๑) Springer Science+Business Media, LLC, part of Springer Nature 2020

\begin{abstract}
Acute viral pneumonia, hypoxemic respiratory failure and severe inflammatory response are hallmarks of severe coronavirus disease 2019 (COVID-19). The COVID-19-associated inflammatory state may further lead to symptomatic thromboembolic complications despite prophylaxis. We report a 66-year-old female patient with post-mortem diagnosis of COVID-19 who presented progressive livedo racemosa, acute renal failure and myocardial injury, as well as an absence of respiratory symptoms. Transthoracic echocardiography showed severe spontaneous echo contrast in the right cardiac chambers and right-sided cardiac overload presumed to result from pulmonary microvascular thrombosis or embolism. D-dimer levels were increased. The patient developed an acute ischemic stroke and died 2 days following presentation despite therapeutic anticoagulation. Her predominantly thromboembolic presentation supports the concept of coronavirus infection of endothelial cells and hypercoagulability, or COVID-19 endotheliitis. The case we report highlights that COVID-19-associated hyperacute multiorgan thromboembolic storm may precede or present disproportionately to respiratory involvement.
\end{abstract}

Keywords COVID-19 $\cdot$ Thromboembolism $\cdot$ Spontaneous echo contrast

\section{Highlights}

- Thromboembolic complications of COVID-19 may precede respiratory symptoms.

- Direct endothelial cell infection and hypercoagulability may cause COVID-19-associated thromboembolisms.

- Multi-organ thromboembolisms may occur despite therapeutic anticoagulation.

Electronic supplementary material The online version of this article (https://doi.org/10.1007/s11239-020-02173-w) contains supplementary material, which is available to authorized users.

Sylvain Lanthier

sylanthier@gmail.com

Faculty of Medicine, McGill University, Montreal, Canada

2 Department of Medicine, University of Montreal, Montreal, Canada

3 Department of Medicine, University of Ottawa, Ottawa, Canada

4 Department of Neurosciences, University of Montreal, Montreal, Canada
- Future studies should prospectively assess thromboembolic prophylaxis and treatments in COVID-19.

\section{Introduction}

Acute viral pneumonia, hypoxemic respiratory failure and a marked inflammatory response are hallmarks of severe coronavirus disease 2019 (COVID-19). Up to 50\% of critically ill patients may develop symptomatic thromboembolic complications despite prophylaxis [1], reflecting the interplay between inflammation and thrombosis [2]. We report a patient whose predominantly thromboembolic manifestations support the concept of coronavirus infection of endothelial cells and hypercoagulability (COVID-19 endotheliitis) [3].

\section{Case report}

A 66-year-old woman presented for pre-syncope. She previously had symptomatic junctional bradycardia and a permanent pacemaker implanted 6 months earlier. Her past 
medical history included immune thrombocytopenia in 2013 , breast cancer surgery and chemotherapy in 2011, and splenectomy and radiotherapy following a Hodgkin's lymphoma diagnosis in 1970. She was also known for a 3-year history of isolated Raynaud's phenomenon, with negative antinuclear and extractable nuclear antigen antibodies and slightly positive rheumatoid factor $(44 \mathrm{IU} / \mathrm{mL})$. Her medication included long-term use of tamoxifen, and aspirin for non-severe coronary artery disease.

She described generalized malaise for a few hours preceding her pre-syncopal episode but denied any infectious symptoms, including dyspnea and cough. Blood pressure was at $170 / 80 \mathrm{mmHg}$, heart rate at $90 / \mathrm{min}$, saturation at $93 \%$ on room air and oral temperature at $37.6{ }^{\circ} \mathrm{C}$. Other than mild livedo, physical exam was unremarkable. Initial workup (Table 1) showed elevated white blood cell count, creatinine and hs-troponin T levels. COVID-19 PCR was negative. Electrocardiogram showed normal sinus rhythm. Pacemaker tested functional. Lung X-ray showed a small retrocardiac infiltrate, suspicious of infection (Fig. 1). Aspirin was continued and she was started on piperacillin-tazobactam and prophylactic anticoagulation with dalteparin (5000 IU daily).

The next day, she remained clinically stable. Laboratory test results, however, deteriorated (Table 1). Transthoracic echocardiography showed very unusual hyperdense smoke-like haze in the right cardiac chambers and the inferior vena cava (Fig. 2a, b; Electronic supplementary materials: Videos 1 to 3). Mild hypokinesia of the right ventricle, abnormal interventricular septal D-shape flattening (Fig. 2c, Electronic supplementary materials: Video 4) and elevated tricuspid regurgitation jet velocity (Fig. 2d) indicated increased pulmonary artery pressure. There was

Table 1 Blood test results

\begin{tabular}{llll}
\hline & $\begin{array}{l}\text { Day 0 } \\
\text { (admis- } \\
\text { sion) }\end{array}$ & Day 1 & Day 2 (death) \\
\hline WBC $\left(\times 10^{9} / \mathrm{L}\right)$ (neutrophils) & $\begin{array}{l}25.2 \\
(58 \%)\end{array}$ & $\begin{array}{l}21.9 \\
(54 \%)\end{array}$ & 24.0 \\
& 117 & 92 & 102 \\
Hemoglobin $(\mathrm{g} / \mathrm{L})$ & 148 & 169 & 195 \\
Platelets $\left(\times 10^{9} / \mathrm{L}\right)$ & 136 & 186 & 158 \\
Creatinine $(\mu \mathrm{mol} / \mathrm{L})$ & 210 & 303 & \\
Creatine phosphokinase $(\mathrm{U} / \mathrm{L})$ & 935 & \\
Hs-troponins $(\mathrm{ng} / \mathrm{L} ;$ normal $<14)$ & 247 & 428 & \\
ALT $(\mathrm{U} / \mathrm{L})$ & & 111 & \\
GGT $(\mathrm{U} / \mathrm{L})$ & & 62 & $>4400$ \\
Total bilirubin $(\mu \mathrm{mol} / \mathrm{L})$ & & & 11748 \\
D-dimers $(\mu \mathrm{g} / \mathrm{L})$ & & & \\
Ferritin $(\mu \mathrm{g} / \mathrm{L})$ & & & \\
\hline
\end{tabular}

$W B C$ white blood cells, $A L T$ alanine aminotransferate, GGT gammaglutamyl transferase

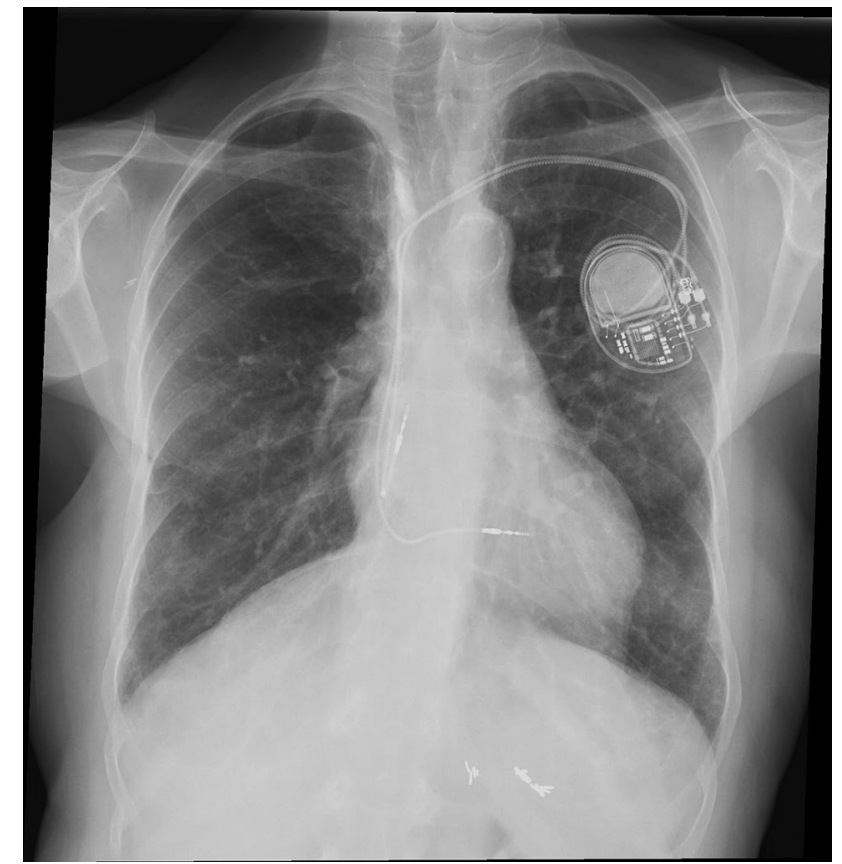

Fig. 1 Lung X-ray: Retrocardiac infiltrate

no clinical evidence of deep vein thrombosis. Therapeutic enoxaparin anticoagulation was started (1 mg/kg BID).

Last-seen neurologically normal at 4:00 AM on Day 2 , she was found with expressive aphasia and rightsided hemiparesis at 7:00 AM. Blood pressure was at $110 / 50 \mathrm{mmHg}$, heart rate at $60 / \mathrm{min}$ and saturation at $97 \%$ on room air. Skin showed diffuse livedo racemosa, involving the whole body at this time. Head CT documented loss of left frontal lobe gray-white matter differentiation, consistent with a hyperacute infarct. Full dose anticoagulation contraindicated thrombolytic therapy. Laboratory investigations showed increased D-dimer and ferritin levels (Table 1). Considering suspected hypercoagulability in the context of the COVID-19 pandemic, therapeutic anticoagulation was maintained but transitioned from enoxaparin to intravenous unfractionated heparin despite the risk of hemorrhagic transformation. Within minutes of starting heparin without bolus, there was hemodynamic collapse (mean arterial pressure of $60 \mathrm{mmHg}$ ). Heparin was discontinued. A blood sample was sent to the coagulation lab. Blood glucose was at $3.1 \mathrm{mmol} / \mathrm{L}$ and lactates at $6 \mathrm{mmol} / \mathrm{L}$. Cardiorespiratory arrest occurred at 9:42 AM. Resuscitation maneuvers were not performed, following the patient's wishes. Coagulation and antiphospholipid antibody tests were cancelled due to a strongly hemolyzed and "unreadable" blood sample. Post-mortem COVID-19 PCR returned positive. 
Fig. 2 Transthoracic echocardiography: spontaneous contrast in the right cardiac chambers (a) and the inferior vena cava (b), abnormal interventricular septal D-shape flattening (c) and elevated tricuspid regurgitation jet velocity (d). $R V$ right ventricle, $R A$ right atrium, $L A$ left atrium, $I V C$ inferior vena cava, $L V$ left ventricle, $T R$ tricuspid regurgitation, $P A P$ pulmonary artery pressure
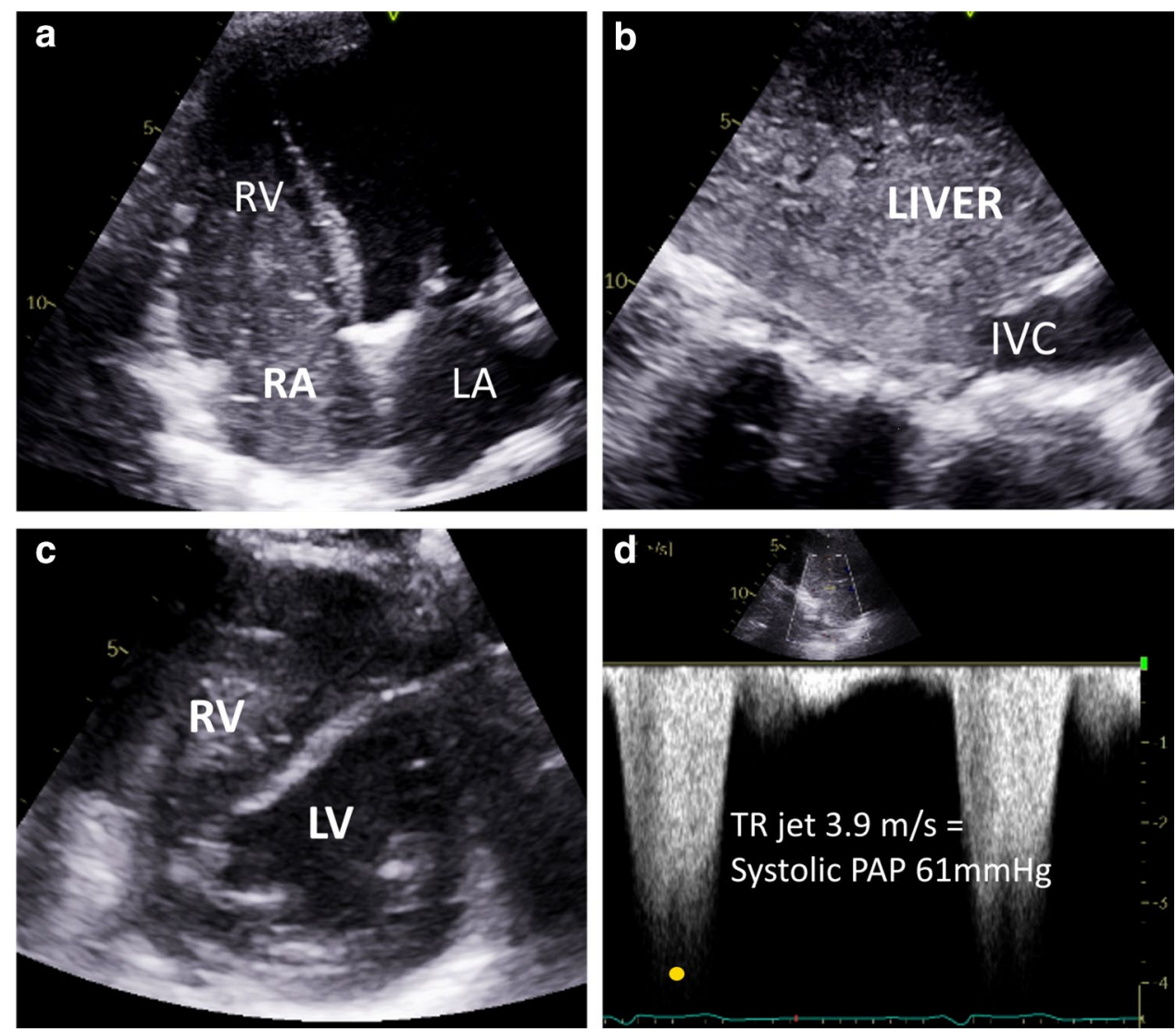

\section{Discussion}

The COVID-19 patient we report had evidence of multiorgan damage at presentation, and no infectious symptoms. As she rapidly deteriorated, a fulminant thromboembolic process was suspected. She presented right-sided cardiac overload likely secondary to pulmonary embolism or extensive microvascular thrombosis, livedo racemosa, and possibly kidney, muscle and liver ischemia. This thromboembolic storm culminated in an ischemic stroke, despite a combination of aspirin and therapeutic doses of anticoagulants, and cardiorespiratory arrest on day 2 . This clinical course together with increased D-dimer levels and a positive COVID-19 PCR is consistent with the recent concept of COVID-19 endotheliitis, which involves direct coronaviral binding to the endothelial cell angiotensin-converting enzyme 2 receptor, endothelial dysfunction, and hypercoagulability [3]. COVID-19 is therefore a thrombo-inflammatory condition [2], in which the prothrombotic component may occasionally precede or predominate over respiratory involvement. Other authors have reported COVID-19 patients with single-organ thromboembolic presentation without viral symptoms (https://www.medrxiv.org/conte nt/10.1101/2020.05.03.20077206v2, accessed May 20, 2020), but coexistent or covert causes other than hypercoagulability cannot be excluded.
COVID-19-associated hypercoagulability may also involve other mechanisms such as complement-mediated endothelial injury [4], cytokine-induced systemic inflammatory response [5], antiphospholipid antibodies [6], and vascular stasis. However, the patient we report had stable platelet counts, excluding disseminated intravascular coagulation and heparin-induced thrombocytopenia. The 9-year interval without evidence of recurrent breast cancer reduces the likelihood of a paraneoplastic process.

The remarkable aspect of dense intracavitary spontaneous echo contrast on echocardiography points toward blood stagnation, and possibly hyperfibrinogenemia (not measured herein) [7]. Such a finding was also reported with cold agglutinin disease [8] and immune-mediated hemolytic anemia [9]. In the present clinical context, the signs of right ventricular pressure overload and dysfunction also strongly suggest pulmonary thromboembolism.

The patient we report had increased D-dimer levels. She experienced a rapidly fatal thromboembolic storm despite aspirin and anticoagulant treatments. High D-dimer levels reflect excessive coagulation and fibrinolysis, and predict venous thromboembolism [10] and mortality [11] in patients hospitalized with COVID-19. Anticoagulant treatment seems to improve in-hospital outcomes [12], but the optimal dosing and duration remain undetermined 
for COVID-19 patients, and particularly for those with increased D-dimer levels [5, 10].

\section{Conclusion}

In the current report, a patient with post-mortem diagnosis of COVID-19 presented progressive livedo racemosa, acute renal failure and myocardial injury, and no respiratory symptoms. Extremely severe spontaneous echo contrast was shown in the right cardiac chambers, as well as right ventricular pressure overload and dysfunction due to presumed pulmonary thromboembolisms. She had an ischemic stroke and died 2 days after presentation. COVID-19-associated hypercoagulability and thromboembolisms may thus precede or present disproportionately to respiratory involvement, which is consistent with the concept of COVID-19 endotheliitis [3].

Author contributions SC: data acquisition, literature revision, manuscript writing. GG, FP and PT: manuscript writing, critical revision of manuscript for intellectual content related to cardiology; MG: manuscript writing, critical revision of manuscript for intellectual content related to internal medicine; MC: critical revision of manuscript for intellectual content related to hematology; SL: literature revision, manuscript writing and critical revision for intellectual content, supervision.

Funding Not applicable.

Data availability Not applicable.

\section{Compliance with ethical standards}

Conflicts of interest The authors declare that they have no conflict of interest.

Ethics approval Not applicable to case reports.

Consent to participate Not applicable.

Consent for publication The patient's sister (closest relative) provided written informed consent to the submission of the case report to the journal.

\section{References}

1. Klok FA, Kruip MJHA, van der Meer NJM et al (2020) Confirmation of the high cumulative incidence of thrombotic complications in critically ill ICU patients with COVID-19: an updated analysis. Thromb Res 191:148-150. https://doi.org/10.1016/j.throm res.2020.04.041

2. Jackson SP, Darbousset R, Schoenwaelder SM (2019) Thromboinflammation: challenges of therapeutically targeting coagulation and other host defense mechanisms. Blood 133:906-918. https:// doi.org/10.1182/blood-2018-11-882993

3. Varga Z, Flammer AJ, Steiger P et al (2020) Endothelial cell infection and endotheliitis in COVID-19. Lancet 395:1417-1418. https ://doi.org/10.1016/S0140-6736(20)30937-5

4. Magro C, Mulvey JJ, Berlin D et al (2020) Complement associated microvascular injury and thrombosis in the pathogenesis of severe COVID-19 infection: a report of five cases. Transl Res. https://doi. org/10.1016/j.trs1.2020.04.007

5. Bikdeli B, Madhavan MV, Jimenez D et al (2020) COVID-19 and Thrombotic or thromboembolic disease: implications for prevention, antithrombotic therapy, and follow-up. J Am Coll Cardiol. https://doi.org/10.1016/j.jacc.2020.04.031

6. Zhang Y, Xiao M, Zhang S et al (2020) Coagulopathy and antiphospholipid antibodies in patients with Covid-19. N Engl J Med 382:e38. https://doi.org/10.1056/NEJMC2007575

7. Rastegar R, Harnick DJ, Weidemann P et al (2003) Spontaneous echo contrast videodensity is flow-related and is dependent on the relative concentrations of fibrinogen and red blood cells. J Am Coll Cardiol 41:603-610. https://doi.org/10.1016/s0735 -1097(02)02898-X

8. Kanyal R, Brugger J, Ramoutar A, Arshad W, Kurbaan AS, Xiao HB (2014) Spontaneous contrast in all cardiac chambers in a patient with a normal heart: case report with literature review. Int J Cardiol 175:e19-e20. https://doi.org/10.1016/j.ijcard.2014.04.234

9. Dogan M, Sari M, Acikel S, Akyel A, Albayrak M, Yeter E (2014) Dense spontaneous echo contrast in the right heart chambers of a patient with autoimmune hemolytic anemia. Herz 39:767-769. https://doi.org/10.1007/s00059-013-3889-9

10. Artifoni M, Danic G, Gautier G et al (2020) Systematic assessment of venous thromboembolism in COVID-19 patients receiving thromboprophylaxis: incidence and role of D-dimer as predictive factors. J Thromb Thrombolysis. https://doi.org/10.1007/ s11239-020-02146-Z

11. Zhang L, Yan X, Fan Q et al (2020) D-dimer levels on admission to predict in-hospital mortality in patients with Covid-19. J Thromb Haemost. https://doi.org/10.1111/jth.14859

12. Paranjpe I, Fuster V, Lala A et al (2020) Association of treatment dose anticoagulation with in-hospital survival among hospitalized patients with COVID-19. J Am Coll Cardiol. https://doi. org/10.1016/j.jacc.2020.05.001

Publisher's Note Springer Nature remains neutral with regard to jurisdictional claims in published maps and institutional affiliations. 